sists of the set of all $n$ such that $E_{d} n \in T$. The Gödel number of $E_{d}$ is, of course, $d$ and the Gödel number of $n$ (i.e. of a string of 1's of length $n)$ is $2^{n}-1$. Thus $A$ consists of all $n$ such that $d *\left(2^{n}-1\right) \in T_{0}$. We add to $\left(S_{2}\right)$ :

Axiom. $P 1-11$.

Production. $P x-y \rightarrow P x 1-y y$.

$P$ represents the set of all ordered pairs $(i, j)$ such that $2^{i}=j$.

Then we add:

Production. $P x-y 1, C d-y-z, T_{0} z \rightarrow Q x$.

In this system (S), " $Q$ " represents $A$.

\title{
REFERENCES
}

1. E. Post, Formal reductions of the general combinatorial decision problem, Amer. J. Math. vol. 65 (1943) pp. 192-215.

2. P. C. Rosenbloom, The elements of mathematical logic, New York, Dover Publications, Inc., 1950, Chapter IV.

3. R. M. Smullyan, Theory of formal systems, Annals of Mathematics Studies, no. 47, Princeton University Press, 1961.

Princeton University

\section{THE AXIOM FOR CONNECTED SETS}

\section{R. L. STANLEY}

1. Introduction. The basic motivation for this study was a desire to find a genuinely unified postulational principle which incorporated both the Axiom of Choice and the "axiom for sets," which latter means an appropriate analogue of the Aussonderungsaxiom to provide for the existence of sets. The possibility of thus uniting these two axiomatic principles has become especially interesting since adding the Axiom of Choice has been shown to be not only safe, ${ }^{1}$ but necessary as well. ${ }^{2}$ In particular, it was further hoped and expected that such a principle, when found, could be expressed naturally as a membership-equivalence statement-that is, essentially of the form

Presented to the Society, June 20, 1959 under the title The postulate for connected sets; received by the editors April 11, 1960 and, in revised form, May 31, 1960.

${ }^{1}$ Gödel showed [1] that for certain systems of set theory, adding the Axiom of Choice does not bring inconsistency.

${ }^{2}$ Mendelson showed [2] that the Axiom of Choice is independent of the other, usual axioms for set theory, hence is indispensable for developments which employ it essentially. 
' . . $(\exists \beta)(\alpha)(\alpha \in \beta . \equiv \ldots)]$, as are the axioms for sets in various systems.

Such a conceptually unified principle was found, and is presented here. This principle contrasts interestingly with the usual, so-called "Zorn lemmas" in two marked ways. First, it is conceptually much simpler than the lemmas, since it incorporates only the very "beginning" set-concepts, whereas the lemmas usually must be stated in terms of ideas which are defined relatively quite a bit later. Second, this principle is obviously and importantly much stronger than any standard Zorn lemma. The usual lemma is equivalent to the Axiom of Choice, once given the context of a well-developed, "pre-Choice" set theory within which to demonstrate that equivalence. The principle at hand, however, yields not only the Axiom of Choice, but also the axiom for sets, thus comprehending within itself the main foundationstone of pre-Choice set theory as well as the usual, post-Choice materials.

2. The axiom. In its classical, or naive form, before any restrictions are installed to prevent logical paradoxes, this axiom is:

$A C S .^{3}$ If $\beta$ is different from $\alpha_{1}$ and $\alpha_{2}$, and is not free in $\phi_{1}$ or $\psi_{12},{ }^{4}$ then

$\vdash^{\mathrm{I}}(R \psi . S \psi . T \psi) \supset(\exists \beta)\left(\alpha_{1}\right)\left(\alpha_{1} \in \beta . \equiv . \phi_{1} .\left(\alpha_{2}\right)\left(\psi_{12}, \alpha_{1} \neq \alpha_{2} . \supset . \alpha_{2} \in \beta\right)\right)^{\mathrm{I}}$.

$\left.{ }^{\top} R \psi\right],[S \psi]$, and $\left[T \psi^{\top}\right.$ mean respectively that $\psi$ is reflexive (for $\alpha$ terms which satisfy condition $\phi$ ), symmetric, and transitive; formally, these are abbreviations of $\left.{ }^{\top}\left(\alpha_{1}\right)\left(\phi_{1} \supset \psi_{11}\right)^{\top}, \Gamma\left(\alpha_{1}\right)\left(\alpha_{2}\right)\left(\psi_{12} \supset \psi_{21}\right)\right]$, and $\Gamma\left(\alpha_{1}\right)\left(\alpha_{2}\right)\left(\alpha_{3}\right)\left(\psi_{12} \cdot \psi_{23} . \supset \psi_{13}\right){ }^{1}$.

This amounts to asserting the following: given any two conditions $\phi_{1}$ and $\psi_{12}$, where $\psi_{12}$ is reflexive for any elements which satisfy $\phi_{1}$,

' ' $A C S$,' of course, denotes this new postulational principle. ' $A C$ ' and ' $A S$ ' will be used to abbreviate 'Axiom of Choice' and 'axiom for sets,' respectively. 'Axiom of Choice' means here Zermelo's familiar, unrestricted principle, conceived in terms of sets, to the effect that, for any set $S$ of nonempty, nonoverlapping, member sets, $s_{i}$, there is an Auswahlmenge, or "choice-set," $A$, whose membership is made up of one and only one member from each such $s_{i}$. $A S$ was characterized in section one above.

${ }^{4}$ In this discussion, $\phi_{i}$ and $\psi_{i j}$, for example, are used to represent the results of validly substituting $\alpha_{i}$ for $\alpha_{1}$ in $\phi$, and $\alpha_{i}$ and $\alpha_{j}$ for $\alpha_{1}$ and $\alpha_{2}$ in $\psi$, respectively. 'Validly' means here that quantifications are to be varied alphabetically, where necessary, to preserve freedom of substituted terms. For example, ' $(x)(y)(x=w . \supset . x=y)^{\prime}$ becomes ' $(z)(y)(z=x . \supset . z=y)$ ', not ' $(x)(y)(x=x . \supset . x=y)$ ', when ' $x$ ' is substituted for ' $w$.' Except where specific departures are thus indicated, notational conventions are the same as those of [3], with small Greek letters representing set-variables, large Greek letters standing for arbitrary formulae, corners acting as quasi-quotation marks, and $|\vdash \phi|$ abbreviating Tthe closure (under universal quantification) of $\phi$ is a theorem l. 
and $\psi_{12}$ is absolutely symmetric and transitive, then there exists (at least) some set $\beta$ whose members individually satisfy the condition $\phi_{1}$, and collectively are connected under the (relational) condition $\sim \psi_{12} ; \beta$ is also "full" or maximal in the sense that adding any more members would falsify one or both of these two circumstances.

Of course, to serve as a postulational principle in any system, $A C S$ must be restricted appropriately to forestall contradictions. Depending on the system, $A C S$ will take various forms.

Where the system restricts all meaningfulness to type-conformant formulae, as does Principia mathematica, ACS will be restricted throughout, both in $\phi$ and $\psi$ jointly, to conform wholly to the canons of types. In that case, $A C S$ will still be equivalent to $(A S \cdot A C), A S$ being type-restricted as usual for such a system, and $A C$ being wholly type-conformant in the first place.

In Zermelo's system of set theory, the appropriate version is

$$
\begin{array}{r}
\mathrm{ACS}_{2} . \vdash^{\top}(\gamma)\left(R \psi . S \psi \cdot T \psi \cdot \supset(\exists \beta)\left(\alpha_{1}\right)\left(\alpha_{1} \in \beta . \equiv . \alpha_{1} \in \gamma . \phi_{1}\right.\right. \\
\left.\left..\left(\alpha_{2}\right)\left(\psi_{12} . \alpha_{1} \neq \alpha_{2} . \supset . \alpha_{2} \tilde{\in} \beta\right)\right)\right)^{\top} .
\end{array}
$$

The equivalence of this form with the conjunction of $A S_{z}$ and $A C$ is conditional upon two more of Zermelo's axioms, those of Summation $(A S m)$ and for the Set of Subsets $(A S b)$. Within the pre-set context of Basal Logic (that is, the propositional calculus, quantification theory, and theory of identity), the following implications can be proven:

$$
\begin{aligned}
\left(A S m . A C S_{z}\right) & \supset\left(A S_{z} . A C\right) ; \\
\left(A S b . A S_{z} . A C\right) & \supset A C S_{z} .
\end{aligned}
$$

Consequently, in a somewhat over-stated, but rather more suggestive form:

$$
(A S m . A S b) \supset\left(A C S_{z} \equiv . A S_{z} . A C\right) .
$$

In Quine's system $N F$, adding $A C$ leads to contradiction, so not only $A S$, but $A C$ also must be restricted in this system. If one considered only the logical paradoxes, the natural restriction for $A C S_{n f}$ would require simply that $\left.{ }^{[}\left(\phi_{1} \cdot \psi_{12}\right)\right]$ be stratified. Such a form would certainly still support the disproof of the unlimited $A C$, however, so further weakening, now in its relational content, appears necessary to prevent $A C S_{n f}$ from yielding the strong $A C$ also, and thereby inconsistency.

Specker derived [4] the negation of the strong axiom of choice as a theorem of Quine's system New foundations. 
3. $A C S \supset A S .6$ Consider the instance of $A C S$ in which $\phi_{1}$ is the appropriate condition of $A S$, and $\psi_{12}$ is $\left[\left(\alpha_{1}=\alpha_{2}\right)\right]$. Since identity is absolutely reflexive, symmetric, and transitive, $A C S$ reduces directly to

$$
\left.\vdash^{\top}(\exists \beta)\left(\alpha_{1}\right)\left(\alpha_{1} \in \beta . \equiv . \phi_{1} \cdot\left(\alpha_{2}\right)\left(\alpha_{1}=\alpha_{2} . \alpha_{1} \neq \alpha_{2} . \supset . \alpha_{2} \tilde{\in} \beta\right)\right)\right] .
$$

Due to its impossible antecedent, the conditional is true for all values of its variables, hence the whole right side of the biconditional becomes equivalent to its part, the formula $\phi_{1}$. This instance of $A C S$ thus collapses into

$$
\vdash^{\lceil}(\exists \beta)\left(\alpha_{1}\right)\left(\alpha_{1} \in \beta . \equiv \phi_{1}\right)^{]},
$$

which is $A S$.

4. $A C S \supset A C$. Consider the instance of $A C S$ in which $\phi_{1}$ is ' $\left(\exists s_{1}\right)\left(\alpha_{1} \in s_{1} . s_{1} \in S\right)$ ', and $\psi_{12}$ is ' $\left(\exists s_{1}\right)\left(\alpha_{1,2} \in s_{1} . s_{1} \in S\right)$ ', with ' $S$ ' denoting the usual set of nonempty sets, in $A C$. Plainly $\phi_{1}$ implies $\psi_{11}$, and furthermore $\psi_{12}$ is symmetric and transitive on $\alpha_{1}$ and $\alpha_{2}$, given $A C$ 's usual hypothesis that members of $S$ are mutually exclusive. With its antecedent thus satisfied, $A C S$ reduces to

$$
\vdash\left\lceil(\exists \beta)\left(\alpha_{1}\right)\left(\alpha_{1} \in \beta . \equiv . \phi_{1} .\left(\alpha_{2}\right)\left(\psi_{12} . \alpha_{1} \neq \alpha_{2} . \supset . \alpha_{2} \tilde{\in} \beta\right)\right)\right] \text {. }
$$

This asserts the existence of some class $\beta$ such that: (1) if any element $\alpha_{1}$ belongs to $\beta$, then $\alpha_{1}$ is a member of some member of $S$, but no other element belongs both to $\beta$ and to any $S$-member. to which $\alpha_{1}$ belongs; and (2) if any $\alpha_{1}$ belongs to some member of $S$, but every other element which belongs to an $S$-member which contains $\alpha_{1}$ is excluded from $\beta$, then $\alpha_{1}$ belongs to $\beta$. Again given that $S$-members are mutually exclusive, this $\beta$ clearly is a set whose membership is built by taking one and only one element from each member of $S$, and such a $\beta$ meets the requirements for the usual Auswahlmenge, whose existence is the consequent in $A C . A C$ thus follows from the assumption of $A C S$.

5. ( $A S . A C) \supset A C S$. If the hypothesis of $A C S$ holds, then its for-

- These demonstrations, sketched quite informally, trace out only the main outlines of the completely rigorous, lengthy, formal proofs. The proofs are "classical" or "natural" in the sense that no restrictions against paradox are incorporated, but neither are any steps taken which employ or yield paradoxical results. Such basic models of axioms and proofs are useful preliminary outlines, to be restricted in any direction and supplemented as necessary to suit any particular system's needs. For example, one such instance system would be type-theoretic; since no steps here violate type-restrictions, one needs merely to preface in general that all proof-lines with unspecified formulae be type-conformant. 
mula $\psi_{12}$ is symmetric and transitive, and reflexive for all $\alpha_{1}$ such that $\phi_{1}$ is true. $A S$ is the main guarantee that a set exists for every specified, predicative condition, and $A C$ is a generalization which is true for all values of ' $S$ '. Given $A S$ and $A C$, then, one may assign to ' $S$ ' the instantial value ' ${ }^{\hat{s}_{1}}\left(\exists \alpha_{1}\right)\left(\phi_{1} . s_{1}=\hat{\alpha}_{1} \phi_{1} \cap \hat{\alpha}_{1} \hat{\alpha}_{2} \psi_{12}{ }^{\prime \prime}\left\llcorner\alpha_{1}\right){ }^{\prime}\right.$. So defined, $S$ consists of members $s_{i}$, whose own members in turn are all the elements $\alpha_{j}$ for which $\phi_{j}$ is true, and which satisfy the relational condition $\psi_{j k}$ with some $\alpha_{k}$ which similarly belongs to $s_{i}$ and satisfies $\phi_{k}$.

For any $s_{1}$ to belong to $S$, there must exist some such $\alpha_{1}$ for which $\phi_{1}$ is true. By the hypothesis of $A C S, \phi_{1}$ implies $\psi_{11}$, so that $\alpha_{1}$ itself belongs to this image-set, and therefore $s_{1}$ contains at least the member $\alpha_{1}$, satisfying the nonemptiness requirement in the hypothesis of $A C$. Furthermore, since $\psi_{12}$ is a symmetric and transitive condition, any $s_{1}$ will consist simply of all the $\alpha_{i}$ for which $\phi_{i}$ holds and which satisfy condition $\psi_{i j}$ with respect to any member of $s_{1}$. If any $s_{1}$ and $s_{2}$ have a common member $\alpha_{1}$, then, they alike consist of all further $\alpha_{i}$ (within $\hat{\alpha}_{1} \phi_{1}$ ) which bear $\psi_{i 1}$ to $\alpha_{1}$, and hence they must be identical. Thus different members of $S$ are mutually exclusive, satisfying the rest of the $A C$-hypothesis.

With the antecedent thus established, the consequent in $A C$ now holds unconditionally, guaranteeing that an Auswahlmenge $A$ exists, containing one and only one member from each of the $s_{i}$-sets, which are themselves the members of $S$. Now since $\phi$ holds while $\psi_{12}$ fails for any $\alpha_{1}$ and $\alpha_{2}$ which belong to different $s_{i}$-sets, $A$ must consist of all and only those elements $\alpha_{1}$ such that $\phi_{1}$ is true, and such that no other $\alpha_{2}$ for which $\psi_{12}$ holds belongs to $A$. These are precisely the conditions, however, which define the set $\beta$ in $A C S$, so such a set $A$ is a suitable, connected set $\beta$ for $A C S$.

In sum, given $A S$ and $A C$, the antecedent of $A C S$ leads to the consequent of $A C S$, so that $(A S . A C) \supset A C S$.

\section{BIBLIOGRAPHY}

1. K. Gödel, The consistency of the axiom of choice and of the generalized continuum hypothesis with the axioms of set theory, second printing, Princeton, Princeton University Press, 1951.

2. Elliott Mendelson, The independence of a weak axiom of choice, J.Symbolic Logic vol. 21 (1956) pp. 350-366.

3. W. V. Quine, Mathematical logic, revised edition, Cambridge, Harvard University Press, 1951.

4. Ernst P. Specker, The axiom of choice in Quine's new foundations for mathematical logic, Proc. Nat. Acad. Sci. U.S.A. vol. 39 (1953) pp. 972-975.

WAShington State University 